\title{
ON THE HAUSDORFF DIMENSION OF SOME GRAPHS
}

\author{
R. DANIEL MAULDIN AND S. C. WILLIAMS
}

ABSTRACT. Consider the functions

$$
W_{b}(x)=\sum_{n=-\infty}^{\infty} b^{-\alpha n}\left[\Phi\left(b^{n} x+\theta_{n}\right)-\Phi\left(\theta_{n}\right)\right],
$$

where $b>1,0<\alpha<1$, each $\theta_{n}$ is an arbitrary number, and $\Phi$ has period one. We show that there is a constant $C>0$ such that if $b$ is large enough, then the Hausdorff dimension of the graph of $W_{b}$ is bounded below by $2-\alpha-(C / \ln b)$. We also show that if a function $f$ is convex Lipschitz of order $\alpha$, then the graph of $f$ has $\sigma$-finite measure with respect to Hausdorff's measure in dimension $2-\alpha$. The convex Lipschitz functions of order $\alpha$ include Zygmund's class $\Lambda_{\alpha}$. Our analysis shows that the graph of the classical van der WaerdenTagaki nowhere differentiable function has $\sigma$-finite measure with respect to $h(t)=t / \ln (1 / t)$.

We consider the Hausdorff dimension of the graphs of various continuous functions. We introduce a new geometric property of a function: convex Lipschitz of some order, and obtain an upper bound on the dimension of a graph with this property. In particular, our analysis includes functions of the form

$$
f_{b}(x)=\sum_{n=0}^{\infty} b^{-\alpha n} \Phi\left(b^{n} x+\theta_{n}\right)
$$

where $0 \leq \theta_{n}<1, b>1,0<\alpha \leq 1$, and $\Phi$ is periodic with period one. For example, we show that the graphs of the van der Waerden-Takagi functions have Hausdorff dimension one. We also give lower bounds on the dimension of graphs of the form

$$
W_{b}(x)=\sum_{n=-\infty}^{\infty} b^{-\alpha n}\left[\Phi\left(b^{n} x+\theta_{n}\right)-\Phi\left(\theta_{n}\right)\right],
$$

where $0<\alpha<1, b>1,0 \leq \theta_{n}<1$, and $\Phi$ has period one. We note that this series converges uniformly on compact sets if $\Phi$ is Lipschitz and bounded. In particular, if $\Phi^{\prime}$ is continuous and $\alpha$ is fixed, then there is a positive constant $C$ such that

$$
2-\alpha-(C / \ln b) \leq \operatorname{dim} f_{b}=\operatorname{dim} W_{b} \leq 2-\alpha,
$$

for sufficiently large $b$.

Received by the editors November 22, 1985.

1980 Mathematics Subject Classification. Primary 28A75; Secondary 42A32.

Key words and phrases. Fractal dimension, Hausdorff dimension, Weierstrass-Mandelbrot functions.

The first author was supported in part by National Science Foundation Grant DMS 8505923. 
The functions of the form $f_{b}$ or $W_{b}$ have a colorful history, and continue to make an appearance in various fields. In 1872, Weierstrass introduced the functions

$$
K(x)=\sum_{n=0}^{\infty} b^{-\alpha n}\left(\cos 2 \pi b^{n} x\right)
$$

and showed that they were nowhere differentiable in certain cases. G. H. Hardy not only showed that $K(x)$ is nowhere differentiable for all $b>1$ and $0<\alpha \leq 1$, but, in addition, obtained some exact results concerning the local Lipschitz order of these functions [5]. Besicovitch and Ursell obtained lower estimates, somewhat similar to ours, on the dimension of graphs of functions which were required to have a large amount of lacunarity [2]. The functions $W_{b}$ and $f_{b}$ treated here do not meet their requirement. Mandelbrot proposed a study of the functions $W_{b}$ with a view to applications and for its intrinsic properties. It has been conjectured that $\operatorname{dim}\left(W_{b}\right)=2-\alpha$, for all $b>1$, in case $\Phi(x)=\cos 2 \pi x[\mathbf{1}, \mathbf{4}, \mathbf{8}]$. The computer studies of Berry and Lewis indicate the complicated behavior of these functions $[\mathbf{1}]$. If each $\theta_{n}=0$, then $W_{b}$ satisfies the functional equation $g(x)=b^{-\alpha} g(b x)$, and $f_{b}$ satisfies the functional equation $g(x)=b^{-\alpha} g(b x)+\Phi(x)$. The addition of the phases eliminates such scaling behavior. Our techniques show that one can nevertheless recover enough scaling to obtain our estimates on the dimension.

Graphs of functions of the form $f_{b}$ also appear as attractors in dynamical systems [7, 9]. Kaplan, Mallet-Paret, and Yorke have obtained exact results on the Lyapunov dimension of some higher dimensional analogues of these functions and have shown the capacity dimension of $K(x)$ is $2-\alpha$ for $b>1,0<\alpha<1$.

Throughout the paper, we will consider functions as graphs. By $\operatorname{dim}(E)$, we mean the Hausdorff dimension of $E$. Our notation mostly follows that of Rogers [10]. Thus, if $h$ is a generalized dimension function, $h-m(E)$ denotes the measure of $E$ with respect to the measure induced by $h$. In particular, if $\alpha$ is a positive number, then $\alpha-m(E)$ denotes the measure of $E$ with respect to $h(x)=x^{\alpha}$. Our first theorem which we offer without proof is useful in reducing the calculation of the dimension of a graph to the complicated part of the function.

THEOREM 1. If $g$ is Lipschitz, then

$$
\operatorname{dim}(f+g)=\operatorname{dim} f .
$$

In particular, $\operatorname{dim} W_{b}=\operatorname{dim} f_{b}$, where

$$
f_{b}:=\sum_{n=0}^{\infty} b^{-\alpha n} \Phi\left(b^{n} x+\theta_{n}\right),
$$

whenever $\Phi$ is bounded and Lipschitz.

Let us mention that without some restriction on $f$ and $g, \operatorname{dim}(f+g)$ may be greater than $\operatorname{dim} f$. This may be seen as follows:

THEOREM 2. $B=\{f \mid \operatorname{dim} f=1\}$ is a dense $G_{\delta}$ subset of $C[0,1]$. Moreover, if $f \in C[0,1]$, then $f=g_{1}-g_{2}$, where $g_{1}$ and $g_{2}$ have dimension 1 .

ProOF. For each $\alpha>1$, let $L_{\alpha}=\{f \in C[0,1] \mid \alpha-m(f)=0\}$. It is easy to check that $L_{\alpha}$ is a $G_{\delta}$ subset of $C[0,1]$ and, of course, every polynomial is in $L_{\alpha}$. Finally, $\bigcap_{n=1}^{\infty} L_{1+1 / n}=B$. 
Now, suppose $f \in C[0,1]$. Since $B$ and $B+f$ are dense $G_{\delta}$ subsets of $C[0,1]$, $B \cap(B+f) \neq \varnothing$. Therefore, $f=g_{1}-g_{2}$, where $g_{1}, g_{2} \in B$. Q.E.D.

REMARK. Theorem 2 shows that almost every function has Hausdorff dimension one. However, we note that almost every function does not have $\sigma$-finite linear measure. This follows from the facts that for almost every $f, f^{-1}(y)$ is uncountable unless $y$ is the maximum or minimum value of $f[3]$ and, on the other hand, if $f$ has $\sigma$-finite measure, then for almost all $y, f^{-1}(y)$ is countable [4, p. 74].

Our next theorem is useful for obtaining upper bounds on the Hausdorff dimension of a graph. Let $\theta$ map $R^{+}$into $R^{+}$.

DEFINITION. A function $f$ is said to be convex Lipschitz of order $\theta$ on an interval $[a, b]$ provided there is a constant $M$ such that if $a \leq x<x+y \leq b$ and $0 \leq \delta \leq 1$, then

$$
|\Delta(x, y, \delta)|:=|f(x+\delta y)-(\delta f(x+y)+(1-\delta) f(x))| \leq M \theta(y) .
$$

We note that if $f$ is in the class $\Lambda_{\alpha}$ described by Zygmund [11], then $f$ is convex Lipschitz of order $x^{\alpha}$. However, the converse is not necessarily true.

THEOREM 3. Let $\theta$ be a continuous map of $R^{+}$into itself such that (1) if $t>0$, $\theta(t)>0$, (2) $\varlimsup_{t \rightarrow 0} t / \theta(t)<\infty$, and (3) $\exists \beta \geq 0$ such that $\lim _{t \rightarrow 0} \theta(c t) / \theta(t)=c^{\beta}$ for all $c>0$. If $f$ is a continuous map on $[0,1]$ which is convex Lipschitz of order $\theta$, then $f$ has $\sigma$-finite $h-m$ measure, where $h(y)=y^{2} / \theta(y)$.

We first set some notation. We will consider the dyadic expansion of numbers in $[0,1]: x={ }_{\varepsilon} \varepsilon_{1} \varepsilon_{2} \varepsilon_{3} \cdots$, and the $n$th approximation: $x_{n}(x)={ }_{.} \varepsilon_{1} \varepsilon_{2} \cdots \varepsilon_{n}$.

PROOF. For each $M_{0} \geq M$, set

$$
\begin{aligned}
& A\left(M_{0}\right)=\{x \in[0,1] \mid \text { for infinitely many } n \\
& \left.\qquad\left|f\left(x_{n}(x)\right)-f\left(x_{n}(x)+2^{-n}\right)\right| \leq M_{0} \theta\left(2^{-n}\right)\right\} .
\end{aligned}
$$

CLAIM 1. $h-m\left(\left.f\right|_{A\left(M_{0}\right)}\right)<+\infty$.

To prove this claim, temporarily fix $m \in \mathbf{N}$. For each $x \in A\left(M_{0}\right)$, let $n(x)$ be the first $n \geq m$ such that

$$
\left|f\left(x_{n}(x)\right)-f\left(x_{n}(x)+2^{-n}\right)\right| \leq M_{0} \theta\left(2^{-n}\right) .
$$

For each $x \in A\left(M_{0}\right)$, let $I(x)=\left[x_{n(x)}(x), x_{n(x)}(x)+2^{-n(x)}\right]$. Note that if $x, y \in$ $A\left(M_{0}\right)$ and $x \neq y$, then either $I(x)=I(y)$ or else $I(x)$ and $I(y)$ are nonoverlapping. Consider $C_{m}=\left\{I(x) \mid x \in A\left(M_{0}\right)\right\}$. Then $C_{m}$ is a cover of $A\left(M_{0}\right)$ by nonoverlapping intervals. Thus,

$$
\bigcup_{x \in A\left(M_{0}\right)} I(x) \times f(I(x)) \supset \operatorname{Graph}\left(\left.f\right|_{A\left(M_{0}\right)}\right)
$$

Now,

$$
\operatorname{diam} f(I(x)) \leq 2 M \theta\left(2^{-n(x)}\right)+M_{0} \theta\left(2^{-n(x)}\right) \leq 3 M_{0} \theta\left(2^{-n(x)}\right) .
$$


Therefore, the rectangle $I(x) \times f(I(x))$ can be covered by $\left(3 M_{0} \theta\left(2^{-n(x)}\right) / 2^{-n(x)}\right)+1$ squares each with edge length $2^{-n(x)}$. Thus, we have

(8)

$$
\begin{aligned}
& (h-m)\left[\left.f\right|_{A\left(M_{0}\right)}\right] \leq \varliminf_{m \rightarrow \infty}\left[\sum_{I(x) \in \mathcal{C}_{m}} 2 \frac{2^{-2 n(x)}}{\theta\left(\sqrt{2} 2^{-n(x)}\right)}\left(3 M_{0} \frac{\theta\left(2^{-n(x)}\right)}{2^{-n(x)}}+1\right)\right] \\
& \quad \leq \varliminf_{m \rightarrow \infty}\left[6 M_{0}\left[\sum 2^{-n(x)} \frac{\theta\left(2^{-n(x)}\right)}{\theta\left(\sqrt{2} 2^{-n(x)}\right)}\right]+\sqrt{2} \sum \frac{\sqrt{2} 2^{-n(x)}}{\theta\left(\sqrt{2} 2^{-n(x)}\right)} 2^{-n(x)}\right] .
\end{aligned}
$$

But, if $t$ is small enough, we have

$$
t / \theta(t) \leq Q<+\infty
$$

and

$$
C_{1} \theta(t) \leq \theta(\sqrt{2} t) \leq C_{2} \theta(t),
$$

for some positive constants $C_{1}$ and $C_{2}$. Therefore,

$$
\begin{aligned}
(h-m)\left(\left.f\right|_{A\left(M_{0}\right)}\right) & \leq \varliminf_{m \rightarrow \infty}\left[\left[6 M_{0} / C_{1}+Q \sqrt{2}\right] \sum 2^{-n(x)}\right] \\
& \leq Q \sqrt{2}+6 M_{0} / C_{1}<+\infty .
\end{aligned}
$$

This completes the proof of Claim 1.

Assume $M_{0}>2 M$. If $x={ }_{1} \varepsilon_{1} \cdots \varepsilon_{n} \cdots \notin A\left(M_{0}\right)$, then $\exists m(x) \in \mathbf{N}$ such that for $n \geq m(x)$,

$$
\left|f\left(x_{n}(x)\right)-f\left(x_{n}(x)+2^{-n}\right)\right|>M_{0} \theta\left(2^{-n}\right) .
$$

Claim 2. $\operatorname{sgn}\left(f\left(x_{n}(x)\right)-f\left(x_{n}(x)+2^{-n}\right)\right)$ is constant for $n \geq m(x)$. Otherwise, we have, for example, setting $x_{n}=x_{n}(x)$

$$
M_{0} \theta\left(2^{-n}\right)<f\left(x_{n}\right)-f\left(x_{n}+2^{-n}\right)
$$

and

$$
-M_{0} \theta\left(2^{-(n+1)}\right)>f\left(x_{n+1}\right)-f\left(x_{n+1}+2^{-(n+1)}\right) .
$$

If $\varepsilon_{n+1}=0$, then $x_{n+1}=x_{n}$ and

$$
\begin{aligned}
f\left(x_{n}\right. & \left.+\frac{1}{2} 2^{-n}\right)-\frac{1}{2}\left(f\left(x_{n}+2^{-n}\right)+f\left(x_{n}\right)\right) \\
& =f\left(x_{n}+\frac{1}{2} 2^{-n}\right)-f\left(x_{n}\right)-\frac{1}{2}\left(f\left(x_{n}+2^{-n}\right)-f\left(x_{n}\right)\right) \\
& =\left(f\left(x_{n+1}+2^{-(n+1)}\right)-f\left(x_{n+1}\right)\right)-\frac{1}{2}\left(f\left(x_{n}+2^{-n}\right)-f\left(x_{n}\right)\right) \\
& >M_{0} \theta\left(2^{-(n+1)}\right)+\left(M_{0} / 2\right) \theta\left(2^{-n}\right)>M \theta\left(2^{-n}\right),
\end{aligned}
$$

a contradiction. The other cases are similar. In other words, if $M_{0}>2 M$ and

$$
\left|f\left((j+1) / 2^{n}\right)-f\left(j / 2^{n}\right)\right|>M_{0} \theta\left(2^{-n}\right),
$$

then $f\left((2 j+1) / 2^{n+1}\right)$ is between $f\left((j+1) / 2^{n}\right)$ and $f\left(j / 2^{n}\right)$. This completes the argument for Claim 2.

Set

$$
B\left(M_{0}, m\right)=\left\{x \in[0,1] \mid \forall n \geq m, f\left(x_{n}(x)+2^{-n}\right)-f\left(x_{n}(x)\right)>M_{0} \theta\left(2^{-n}\right)\right\}
$$


and

(17) $C\left(M_{0}, m\right)=\left\{x \in[0,1] \mid \forall n \geq m, f\left(x_{n}(x)+2^{-n}\right)-f\left(x_{n}(x)\right)<-M_{0} \theta\left(2^{-n}\right)\right\}$.

We have $B\left(M_{0}, m\right) \subseteq B\left(M_{0}, m+1\right)$ and $C\left(M_{0}, m\right) \subseteq C\left(M_{0}, m+1\right)$. Since $M_{0}>$ $2 M$, Claim 2 implies

$$
[0,1] \backslash A\left(M_{0}\right)=\left[\bigcup_{m=0}^{\infty} B\left(M_{0}, m\right) \cup C\left(M_{0}, m\right)\right] .
$$

Fix $m$. We will show that each $\left.f\right|_{B\left(M_{0}, m\right)}$ has finite $h$-measure. For $\varepsilon=\left\langle\varepsilon_{1}\right.$, $\left.\varepsilon_{j}\right\rangle \in\{0,1\}^{j}$ and $k \leq j$, define

$$
I(\varepsilon):=\left[. \varepsilon_{1} \cdots \varepsilon_{j}, . \varepsilon_{1} \cdots \varepsilon_{j}+2^{-j}\right]
$$

and

$$
\Delta_{\varepsilon, k} f:=f\left(. \varepsilon_{1} \cdots \varepsilon_{k}+2^{-k}\right)-f\left(. \varepsilon_{1} \cdots \varepsilon_{k}\right) .
$$

For each $n \geq m$, set

$$
C_{n}=\left\{\varepsilon \in\{0,1\}^{n} \mid \Delta_{\varepsilon, k} f>M_{0} \theta\left(2^{-k}\right), \text { for } m \leq k \leq n\right\} .
$$

Now,

$$
\sum_{\varepsilon \in C_{n+1}} \Delta_{\varepsilon, n+1} f \leq \sum_{\varepsilon \in C_{n}} \Delta_{\varepsilon, n} f .
$$

This follows from the facts that if $\left\langle\varepsilon_{1}, \ldots, \varepsilon_{n}, \delta\right\rangle \in C_{n+1}$, then $\left\langle\varepsilon_{1}, \ldots, \varepsilon_{n}\right\rangle \in C_{n}$; $\Delta_{\varepsilon * 0, n+1} f+\Delta_{\varepsilon * 1, n+1} f=\Delta_{\varepsilon, n} f$; and, by the argument given for Claim 2, all of these differences are positive.

For each $n \geq m$,

$$
\operatorname{Graph}\left(\left.f\right|_{B\left(M_{0}, m\right)}\right) \subseteq \bigcup_{\varepsilon \in C_{n}} I(\varepsilon) \times f(I(\varepsilon))
$$

For each $\varepsilon \in C_{n}$, we need no more than $\left(\operatorname{diam} f(I(\varepsilon)) / 2^{-n}\right)+1$ squares with diameter $\sqrt{2} 2^{-n}$ to cover the rectangle $I(\varepsilon) \times f(I(\varepsilon))$.

Let

$$
\begin{aligned}
T_{n} & =\sum_{\varepsilon \in C_{n}}\left(\operatorname{diam} f(I(\varepsilon)) / 2^{-n}+1\right) h\left(\sqrt{2} 2^{-n}\right) \\
& \leq\left[\sum_{\varepsilon \in C_{n}} \operatorname{diam} f(I(\varepsilon)) \frac{h\left(\sqrt{2} 2^{-n}\right)}{2^{-n}}\right]+\sqrt{2} Q<\infty .
\end{aligned}
$$

Obviously, the $h$-measure of $\left.f\right|_{B\left(M_{0}, m\right)}$ is dominated by $\underline{\lim }_{n \rightarrow \infty} T_{n}$. Since, $\operatorname{diam} f(I(\varepsilon)) \leq \Delta_{\varepsilon, n} f+2 M \theta\left(2^{-n}\right)$,

$$
T_{n} \leq \frac{h\left(\sqrt{2} 2^{-n}\right)}{2^{-n}}\left[\sum_{\varepsilon \in C_{n}} \Delta_{\varepsilon, n} f+2 M \sum_{\varepsilon \in C_{n}} \theta\left(2^{-n}\right)\right]+\sqrt{2} Q .
$$

So, for each $n \geq m$,

$$
T_{n} \leq \frac{h\left(\sqrt{2} 2^{-n}\right)}{2^{-n}}\left[\sum_{\varepsilon \in C_{n}} \Delta_{\varepsilon, n} f\right]+\frac{4 M \theta\left(2^{-n}\right)}{\theta\left(\sqrt{2} 2^{-n}\right)}+\sqrt{2} Q .
$$

Since $\varlimsup \lim h(\sqrt{2} t) / t<\infty$ as $t \rightarrow 0$ and $\lim \theta\left(2^{-n}\right) / \theta\left(\sqrt{2} 2^{-n}\right)=2^{-\beta / 2}$, the $T_{n}$ 's are uniformly bounded. Therefore, $\left.f\right|_{B\left(M_{0}, m\right)}$ has finite $h$-measure. 
THEOREM 4. Let $\Phi(x)=\cos 2 \pi x$. If $0<\alpha<1$, then $W_{b}$ is Lipschitz of order $\alpha$ and consequently $\operatorname{dim}\left(W_{b}\right) \leq 2-\alpha$ and $W_{b}$ has finite $(2-\alpha)$-measure. If $\alpha=1$, then $f_{b}$ is not necessarily Lipschitz, but it is always convex Lipschitz of order 1. Consequently, if $\alpha=1, \operatorname{dim} f_{b}=1$ and $f_{b}$ has $\sigma$-finite measure with respect to linear Hausdorff measure in $\mathbf{R}^{2}$.

If $0<\alpha<1$, it is easy to see that $W_{b}$ is Lipschitz of order $\alpha$. The first statement follows from [2]. The last two statements of this theorem follow from Theorem 3 and Hardy's result that if $\alpha=1$ and each $\theta_{n}=0, f_{b}$ is nowhere differentiable [5], and therefore cannot be Lipschitz of order 1 . We will generalize the results of Theorem 4 in Theorems 6 and 7. We also note that if $f$ is convex Lipschitz of order 1 , then $f$ is in Zygmund's class $\Lambda_{*}$.

THEOREM 5. Let $f$ be continuous on an open interval $J$. Then $f$ is convex Lipschitz of order 1 on $J$ if and only if $f$ is in Zygmund's class $\Lambda_{*}$ on $J$.

ProOF. It is easy to check that if $f$ is convex Lipschitz on $J$, then $f$ is in $\Lambda_{*}$.

For the converse, let $M$ be such that $\left|\Delta^{2}(x, h)\right| \leq M h$, where $\Delta^{2}(x, h):=$ $f(x+h)+f(x-h)-2 f(x)$. Fix $x, x+y \in J, x<x+y$. Define an auxiliary function $g$ on $[0,1]$ by $g(\delta):=\Delta(x, y, \delta)$. To prove $f$ is convex Lipschitz it suffices to show $|g(\delta)| \leq M y$ for all $\delta$ in $[0,1]$. Now, $|g(1 / 2)| \leq \Delta^{2}(x+y / 2, y / 2) / 2 \leq M y / 4$. For each $n$, set $D_{n}=\left\{(2 j+1) / 2^{n} \mid 0 \leq j \leq 2^{n-1}-1\right\}$. Since $f \in \Lambda_{*}$, we have

$$
\left|g\left(\frac{2 j+1}{2^{n+1}}\right)\right| \leq \frac{1}{2}\left|g\left(\frac{j}{2^{n}}\right)\right|+\frac{1}{2}\left|g\left(\frac{j+1}{2^{n}}\right)\right|+\frac{M y}{2 \cdot 2^{n+1}},
$$

or

$$
\left|g\left(\frac{2 j+1}{2^{n+1}}\right)\right| \leq \sup \left\{|g(d)| \mid d \in \bigcup\left\{D_{k} \mid k \leq n\right\}\right\}+\frac{M y}{2 \cdot 2^{n+1}} .
$$

By induction, we obtain

$$
\sup \left\{|g(d)| \mid d \in D_{n}\right\} \leq M y\left(1 / 2^{2}+\cdots+1 / 2^{n+1}\right) .
$$

Since $g$ is continuous, $|g(\delta)| \leq M y$ for all $\delta \in[0,1]$. Q.E.D.

THEOREM 6. Suppose $\Phi: R \rightarrow R$ is bounded and convex Lipschitz of order 1 . If $b>1,0<\alpha<1$, and

$$
f_{b}(x)=\sum_{n=0}^{\infty} b^{-\alpha n} \Phi\left(b^{n} x+\theta_{n}\right),
$$

then $f_{b}$ is convex Lipschitz of order $\alpha$. Consequently,

$$
\operatorname{dim}\left(f_{b}\right) \leq 2-\alpha .
$$

Proof. Fix $b>1$ and set $a=b^{-\alpha}$ and $f=f_{b}$. We have

$$
\begin{aligned}
&|\Delta(x, y, \delta)| \leq \mid \sum_{p=0}^{n} a^{p}\left[\Phi\left(b^{p} x+\delta b^{p} y+\theta_{p}\right)-\Phi\left(b^{p} x+\theta_{p}\right)\right. \\
&\left.-\delta\left(\Phi\left(b^{p} x+b^{p} y+\theta_{p}\right)-\Phi\left(b^{p} x+\theta_{p}\right)\right)\right] \mid \\
&+3\|\Phi\| \sum_{p=n+1}^{\infty} a^{p} .
\end{aligned}
$$


So, letting $M$ be a convex Lipschitz constant for $\Phi$,

$$
|\Delta(x, y, \delta)| \leq M y \sum_{p=0}^{n}(a b)^{p}+3\|\Phi\| a^{n+1} /(1-a) .
$$

Thus,

$$
|\Delta(x, y, \delta)| \leq M y(a b)^{n+1} /(a b-1)+3\|\Phi\| a^{n+1} /(1-a) .
$$

Choose $n$ such that $b^{-(n+1)} \leq y<b^{-n}$. Then $a^{n+1}=\left(b^{-(n+1)}\right)^{\alpha} \leq y^{\alpha}$. So,

$$
|\Delta(x, y, \delta)| \leq(M b /(a b-1)+3\|\Phi\| /(1-a)) y^{\alpha} .
$$

Thus, $f$ is convex Lipschitz of order $\alpha$. Q.E.D.

REMARK. If each $\theta_{n}=0$ in equation (27), then $f_{b}$ is the unique bounded solution of the functional equation $f(x)=b^{-\alpha} f(b x)+\Phi(x)$. Moreover, there are $2^{c}$ solutions of this functional equation, and the dimension of a solution can be any number in $[2-\alpha, 2]$.

The functions considered in the next theorem include the van der WaerdenTagaki functions. (Set $\Phi(x)=\operatorname{dist}(x, Z)$.)

THEOREM 7. Consider

$$
f_{b}(x)=\sum_{n=0}^{\infty} b^{-n} \Phi\left(b^{n} x+\theta_{n}\right),
$$

where $b>1$. If $\Phi$ is a bounded Lipschitz continuous function on $R$, then $f_{b}$ has $\sigma$-finite $h$-measure where $h(y)=y / \ln (1 / y)$ and each $f_{b}$ has Hausdorff dimension one. If $\Phi: R \rightarrow R$ is bounded, absolutely continuous and $\Phi^{\prime}$ is Lipschitz, then $f_{b}$ has $\sigma$-finite linear measure.

ProOF. Set $a=b^{-1}$. If $\Phi$ is bounded and Lipschitz, it follows from inequality (28) that

$$
|\Delta(x, y, \delta)| \leq 2\left\|\Phi^{\prime}\right\| n \delta y+3\|\Phi\| a^{n+1} /(1-a)
$$

for all $n$.

If $b^{-(n+1)} \leq y<b^{-n}$, then $n<\ln (1 / y) / \ln b$. So,

$$
|\Delta(x, y, \delta)| \leq\left(2\left\|\Phi^{\prime}\right\| / \ln b\right) y \ln (1 / y)+(3 b\|\Phi\| /(b-1)) y .
$$

Thus, there is a constant $D$ such that

$$
|\Delta(x, y, \delta)| \leq D y \ln (1 / y)
$$

Therefore, by Theorem $3, f_{b}$ has $\sigma$-finite measure with respect to $h(y)=y / \ln (1 / y)$. This implies that the graph of $f$ has Hausdorff dimension one.

Now, if $\Phi$ is bounded, absolutely continuous and $\Phi^{\prime}$ is Lipschitz, it follows from (28) that

$$
\begin{aligned}
|\Delta(x, y, \delta)| \leq & \left|\sum_{p=0}^{n} a^{p}\left[\delta b^{p} y \int_{0}^{1} \Phi^{\prime}\left(b^{p} x+\delta b^{p} y t+\theta_{p}\right)-\Phi^{\prime}\left(b^{p} x+b^{p} y t+\theta_{p}\right) d t\right]\right| \\
& +3\|\Phi\| a^{n+1} /(1-a) .
\end{aligned}
$$


Choose $B$ such that $\left|\Phi^{\prime}(u)-\Phi^{\prime}(v)\right| \leq B|u-v|$. Thus,

$$
\begin{aligned}
|\Delta(x, y, \delta)| & \leq B \delta y \sum_{p=0}^{n}(a b)^{p} \int_{0}^{1}(1-\delta) b^{p} y t d t+3\|\Phi\| a^{n+1} /(1-a) \\
& \leq \delta(1-\delta)\left(B y^{2}\right)\left(a b^{2}\right)^{n+1} / 2\left(a b^{2}-1\right)+3\|\Phi\| a^{n+1} /(1-a) .
\end{aligned}
$$

Choose $n$ such that $b^{-(n+1)} \leq y<b^{-n}$. Then $y^{2} b^{2 n}<1$, and $a^{n+1}=b^{-(n+1)} \leq y$. So

$$
|\Delta(x, y, \delta)| \leq\left(b^{2} B / 8\left(a b^{2}-1\right)+3\|\Phi\| /(1-a)\right) y .
$$

Thus, $f$ is convex Lipschitz of order 1. Q.E.D.

REMARK. We do not know whether the van der Waerden-Tagaki function has $\sigma$-finite linear measure.

THEOREM 8. Suppose $\Phi: R \rightarrow R$ (1) is nonconstant and continuous, (2) has a piecewise continuous derivative, (3) $\Phi(x+1)=\Phi(x)$, and (4) $\|\Phi\|=1$. Fix $0<\alpha<1$. Let $I$ be a subinterval of $[0,1]$ with length $l>0$ such that (i) $\Phi^{\prime}$ is continuous on $I$ and (ii) inf $\Phi^{\prime} \geq \varepsilon>0$ on $I$. There is a constant $C>0$ such that if $b \geq 3 / l$, then

$$
\operatorname{dim}\left(f_{b}\right) \geq 2-\alpha-C / \ln b
$$

where

$$
f_{b}(x)=\sum_{n=0}^{\infty} b^{-\alpha n} \Phi\left(b^{n} x+\theta_{n}\right),
$$

and $\theta_{0}, \theta_{1}, \theta_{2}, \ldots$ are arbitrary phases.

Theorem 8 follows from Theorem 9 .

THEOREM 9. Assume the hypothesis of Theorem 8. Then there is a constant $C>0$ and a function $G:[3 / l, \infty) \rightarrow R^{+}$such that if $b \geq 3 / l$, then there is a Cantor subset $K$ of $R$ and a probability measure $\nu$ supported on $f_{b} \cap(K \times R)$ such that if $X$ is a square of side $z<b^{-1}$ with sides parallel to the coordinate axes, then

$$
\nu(X) \leq G(b) z^{(2-\alpha)-C / \ln b} .
$$

PROOF. For convenience, we assume $\theta_{0}=0$. Set $f=f_{b}$ and set $r=[b l]-1$. Note that the integer $r \geq 2$. We define a system of intervals $\left\{J_{\sigma} \mid \sigma \in r^{*}\right\}$, where $r^{*}=\bigcup_{n=1}^{\infty}\{1, \ldots, r\}^{n}$, as follows. For each $i, 1 \leq i \leq r$, let $q_{i}$ be the largest integer in the interval $b[I+(i-1)]+\theta_{1}$. Since this interval has length $b l$, the integer $q_{i}-r$ is also in it. Set

$$
J_{i}=\left[\frac{q_{i}-\theta_{1}-r}{b}, \frac{q_{i}-\theta_{1}}{b}\right] .
$$

So, $J_{i} \subset I+(i-1), i=1, \ldots, r$, and $\Phi^{\prime}(x) \geq \varepsilon$ if $x \in J_{i}$.

Suppose $J_{\sigma}$ has been chosen of the form

$$
J_{\sigma}=\left[\frac{q_{\sigma}-\theta_{|\sigma|}-r}{b^{|\sigma|}}, \frac{q_{\sigma}-\theta_{|\sigma|}}{b^{|\sigma|}}\right],
$$

where $q_{\sigma}$ is a positive integer, and $|\sigma|$ denotes the length of the sequence $\sigma$. For each $i \in\{1, \ldots, r\}$, let $q_{\sigma^{*} i}$ be the largest integer in the interval

$$
H_{\sigma^{*} i}=b\left(I+q_{\sigma}-r+i-1-\theta_{|\sigma|}\right)+\theta_{|\sigma|+1} .
$$


Set

$$
J_{\sigma^{*} i}=\left[\frac{q_{\sigma^{*} i}-\theta_{|\sigma|+1}-r}{b^{|\sigma|+1}}, \frac{q_{\sigma^{*} i}-\theta_{|\sigma|+1}}{b^{|\sigma|+1}}\right] .
$$

We note the following facts:

$(J 1)$ For each $n,\left\{J_{\sigma} \mid \sigma \in\{1, \ldots, r\}^{n}\right\}$ is a collection of pairwise disjoint intervals of length $r b^{-n}$.

(J2) For each $\sigma$ and $i=1, \ldots, r$,

$$
\begin{aligned}
J_{\sigma^{*} i} & \subset b^{-|\sigma|}\left(I+q_{\sigma}-r+i-1-\theta_{|\sigma|}\right) \\
& \subset\left[\frac{q_{\sigma}-r+i-1-\theta_{|\sigma|}}{b^{|\sigma|}}, \frac{q_{\sigma}-r+i-\theta_{|\sigma|}}{b^{|\sigma|}}\right] \subset J_{\sigma} .
\end{aligned}
$$

(J3) If $x \in J_{\sigma}$, then $\Phi^{\prime}\left(b^{|\sigma|-1} x+\theta_{|\sigma|-1}\right) \geq \varepsilon$.

Of course, $(J 1)$ follows immediately from the construction. The first inclusion of (J2) follows from the fact that since the interval $H_{\sigma^{*} i}$ has length $b l>r+1$, $q_{\sigma^{*} i}-r \in H_{\sigma^{*} i}$. The second inclusion follows from $I \subset[0,1]$. The last inclusion is obvious. Fact (J3) follows from the construction of $J_{\sigma}$.

Let

$$
K=\bigcap_{n=1}^{\infty}\left[\bigcup_{|\sigma|=n} J_{\sigma}\right]
$$

let $\tilde{\nu}$ be the unique probability measure supported on $K$ defined by the condition $\tilde{\nu}\left(J_{\sigma}\right)=r^{-|\sigma|}$, and let $\nu$ be the probability measure supported on $\operatorname{Graph}\left(\left.f\right|_{K}\right)$ defined by

$$
\int_{R^{2}} g(x, y) d \nu(x, y):=\int_{R} g(x, f(x)) d \tilde{\nu}(x),
$$

for $g \in C_{0}\left(R^{2}\right)$. It can be checked that $\nu$ also satisfies

$$
\int_{R^{2}} g(x, y) d \nu(x, y)=\lim _{n \rightarrow \infty} \frac{1}{r^{n}} \sum_{|\sigma|=n} g\left(x_{\sigma}, f\left(x_{\sigma}\right)\right),
$$

where $x_{\sigma}=\inf \left(J_{\sigma}\right)$.

Let $X$ be a square $\left[x_{0}, x_{0}+z\right] \times\left[y_{0}, y_{0}+z\right]$ with $z<b^{-1}$. Let $n$ be the positive integer such that

$$
b^{-(n+1)} \leq z<b^{-n}
$$

and let $k$ be the positive integer such that

$$
b^{-\alpha(n+k)} \leq z<b^{-\alpha(n+k-1)} .
$$

For each $s \in\{0,1, \ldots, k-1\}$, let

$$
\# s=\operatorname{card}\left\{\sigma|| \sigma \mid=n+s \text { and }\left.f\right|_{J_{\sigma}} \cap X \neq \varnothing\right\} .
$$

We have

$$
\nu(X)=\int_{K} 1_{X}(t, f(t)) d \tilde{\nu}(t) \leq \#(k-1) r^{-(n+k-1)} .
$$

Our next task is to obtain some bounds on the size of \#s. 
Suppose $p=n+s,|\sigma|=p$, and $\left.f\right|_{J_{\sigma}} \cap X \neq \varnothing$. Let

$$
g(x)=\sum_{m=0}^{p-1} b^{-\alpha m} \Phi\left(b^{m} x+\theta_{m}\right) .
$$

We have

$$
\|f-g\| \leq \sum_{m=p}^{\infty} b^{-\alpha m}=b^{-\alpha p} /\left(1-b^{-\alpha}\right) .
$$

If $(x, f(x)) \in X$, then

$$
\left[g(x)-b^{-\alpha p} /\left(1-b^{-\alpha}\right), g(x)+b^{-\alpha p} /\left(1-b^{-\alpha}\right)\right] \cap\left[y_{0}, y_{0}+z\right] \neq \varnothing .
$$

If $x \in J_{\sigma}$, then from $(J 3)$, we get

$$
g^{\prime}(x)=\sum_{m=0}^{p-1} b^{(1-\alpha) m} \Phi^{\prime}\left(b^{m} x+\theta_{m}\right) \geq \varepsilon\left(\frac{b^{(1-\alpha) p}-1}{b^{(1-\alpha)}-1}\right) .
$$

In particular, $g$ is increasing on $J_{\sigma}$. Let

$$
E=\left\{x \in J_{\sigma} \mid \text { eq. (44) holds }\right\} .
$$

Thus, $E$ is an interval and if $\left.f\right|_{J_{\sigma^{*} i}} \cap X \neq \varnothing$, then $J_{\sigma^{*} i} \cap E \neq \varnothing$. But $J_{\sigma^{*} i} \subset$ $\left[\left(q_{\sigma}-r+i-1-\theta_{p}\right) / b^{p},\left(q_{\sigma}-r+i-\theta_{p}\right) / b^{p}\right]$ for $i=1, \ldots, r$. Since these last intervals are nonoverlapping, $E$ can meet at most $2+b^{p} \lambda(E)$ of them, where $\lambda$ is Lebesgue measure. So,

$$
\operatorname{card}\left\{i \in\{1, \ldots, r\}|f|_{J_{\sigma^{*}} i} \cap X \neq \varnothing\right\} \leq 2+b^{p} \lambda(E) .
$$

Consequently,

$$
\#(s+1) \leq\left[2+b^{n+s}(m(n+s))\right] \# s,
$$

where $m(n+s)$ is the maximum possible length of $E$ on level $n+s$. Now, $m(n+s) \leq$ $c / d$, where $c$ is the height of the box that $g$ must be in if $f$ is in $X$ on $J_{\sigma}$ and $d=\min \left\{g^{\prime}(x) \mid x \in J_{\sigma}\right\}$. Now, if $(x, f(x)) \in X$, then $(x, g(x))$ is in a box of height $z+2 b^{-\alpha p} /\left(1-b^{-\alpha}\right)$. Since $z<b^{-\alpha(n+k-1)}$ and $p \leq n+k-1$, we have $z<b^{-\alpha p}<b^{-\alpha p} /\left(1-b^{-\alpha}\right)$. So,

$$
m(n+s)<\frac{3}{\varepsilon}\left(\frac{b^{-\alpha p}}{1-b^{-\alpha}}\right)\left(\frac{b^{1-\alpha}-1}{b^{(1-\alpha) p}-1}\right) .
$$

Consider

$$
\begin{aligned}
2+b^{p} & \frac{3}{\varepsilon} \frac{b^{-\alpha p}}{1-b^{-\alpha}} \frac{b^{1-\alpha}-1}{b^{(1-\alpha) p}-1} \\
& =b^{1-\alpha}\left\{\frac{2}{b^{1-\alpha}}+\frac{3}{\varepsilon}\left(\frac{1}{1-b^{-\alpha}}\right)\left(\frac{1-b^{-(1-\alpha)}}{1-b^{-(1-\alpha) p}}\right)\right\} .
\end{aligned}
$$

Consider the factor in \{\} as a function, $h_{p}(b)$. Note that for all $p \geq 1, h_{p}(b) \leq$ $h_{1}(b)$ for $b \geq 3 / l$. Now, $h_{1}(b)$ is continuous and $\lim h_{1}(b)=3 / \varepsilon$ as $b \rightarrow \infty$. Let $\delta=\max \left\{h_{1}(b) \mid b \geq 3 / l\right\} ; 3 / \varepsilon \leq \delta<\infty$. Therefore, from (47), we obtain

$$
\#(s+1) \leq \delta b^{1-\alpha} \# s \text {. }
$$


Since $\# 0 \leq 2$, we find by recursion on $(51)$

$$
\#(k-1) \leq 2 \delta^{k-1} b^{(1-\alpha) k-1} .
$$

Therefore, from (41)

$$
\nu(X) \leq 2 \delta^{k-1} b^{(1-\alpha)(k-1)} r^{-(n+k-1)} .
$$

From (38) and (39),

$$
n(1-\alpha) / \alpha \leq k \leq n(1-\alpha) / \alpha+1+1 / \alpha .
$$

Also, $r^{-1}<2 / l b$. Thus,

$$
\nu(X) \leq 2 \delta^{1 / \alpha} \delta^{n(1-\alpha) / \alpha} b^{-\alpha(k-1)}(2 / l)^{n+k-1} b^{-n},
$$

or,

$$
\begin{aligned}
\nu(X) & \leq 2 \delta^{1 / \alpha} \delta^{n(1-\alpha) / \alpha}(2 / l)^{(n+1) / \alpha} b^{-\alpha(n(1-\alpha) / \alpha-1)} b^{-n} \\
& \leq 2(2 \delta / l)^{1 / \alpha} b^{\alpha}\left[\left(2 \delta^{1-\alpha} / l\right)^{1 / \alpha}\right]^{n} b^{-n(2-\alpha)} .
\end{aligned}
$$

Set $A=2(2 \delta / l)^{1 / \alpha}$ and $B=\left(2 \delta^{1-\alpha} / l\right)^{1 / \alpha}$. Since $b z \geq b^{-n}$,

$$
\nu(X) \leq A b^{2} B^{n} z^{2-\alpha} .
$$

Finally, since $n<-\ln z / \ln b, B^{n}=e^{n \ln B}<z^{-\ln B / \ln b}$. Set $C=\ln B$. Let $G(b)=A b^{2}$. We have

$$
\nu(X) \leq G(b) z^{2-\alpha-C / \ln b} .
$$

To see that $C>0$, it suffices to show that $2 \delta^{1-\alpha} / l>1$. Since $\|\Phi\|=1, \varepsilon l \leq 2$. Since $\delta \geq 3 / \varepsilon$, we have $2 \delta^{1-\alpha} / l \geq 3^{1-\alpha}(2 / l)^{\alpha}$. Thus, $2 \delta^{1-\alpha} / l>1$, for $0<\alpha<1$.

\section{REFERENCES}

1. M. V. Berry and Z. V. Lewis, On the Weierstrass-Mandelbrot fractal functions, Proc. Roy. Soc. London Ser. A 370 (1980), 459-484.

2. A. S. Besicovitch and H. D. Ursell, Sets of fractional dimensions, V: On dimensional numbers of some continuous curves, J. London Math. Soc. (2) 32 (1937), 142-153.

3. A. Bruckner and K. Garg, The level set structure of a residual set of continuous functions, Trans. Amer. Math. Soc. 232 (1977), 307-321.

4. K. J. Falconer, The geometry of fractal sets, Cambridge Tracts in Math., no. 85, Cambridge Univ. Press, Cambridge, 1985.

5. G. H. Hardy, Weierstrass's non differentiable functions, Trans. Amer. Math. Soc. 17 (1916), 301-325.

6. F. Hausdorf, Dimension and ausseres Mass, Math. Ann. 79 (1919), 157-179.

7. James L. Kaplan, John Mallet-Paret, and James A. Yorke, The Lyapunov dimension of a nowhere differentiable attracting torus, Ergodic Theory Dynamical Systems 4 (1984), 261-281.

8. B. B. Mandelbrot, Fractals: Form, chance and dimension, Freeman, San Francisco, Calif., 1977.

9. J. Moser, On a theorem of Anosov, J. Differential Equations 5 (1969), 411-440.

10. C. A. Rogers, Hausdorff measures, Cambridge Univ. Press, Cambridge, 1970.

11. A. Zygmund, Trigonometric series, Cambridge Univ. Press, Cambridge, 1968.

Department of Mathematics, North Texas State University, Denton, Texas 76203-5116

Department of Mathematics, Utah State University, Logan, Utah 84322 